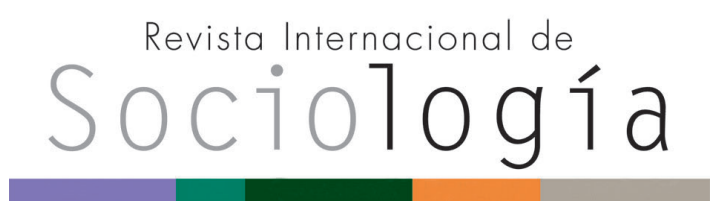

Revista Internacional de Sociología RIS

vol. 76 (1), e081, enero-marzo, 2018, ISSN-L:0034-9712 https://doi.org/10.3989/ris.2018.76.1.16.66

\section{HÁBITOS, REFLEXIÓN Y ESTADOS MENTALES INTENCIONALES}

\author{
Antonio Miguel Camargo \\ Universidad del Norte, Colombia \\ antoniomcamargod@hotmail.com \\ ORCID iD: https://orcid.org/0000-0003-1445-2588
}

Cómo citar este artículo / Citation: Camargo, A. M. 2018. "Hábitos, reflexión y estados mentales intencionales". Revista Internacional de Sociología 76(1): e081. https://doi. org/10.3989/ris.2018.76.1.16.66

\section{HABITS, REFLECTION AND INTENTIONAL MENTAL STATES}

Copyright: () 2018 CSIC. Este es un artículo de acceso abierto distribuido bajo los términos de la licencia de uso y distribución Creative Commons Reconocimiento 4.0 Internacional (CC BY 4.0).

Recibido: 16/04/2016. Aceptado: 26/07/2017. Publicado online: 21/03/2018

\begin{abstract}
Habits and reflection are considered fundamental driving forces of human agency. How they affect our behavior depends on the complex relationships between structure and agent. This article analyzes the role of habits and reflection from the individual, based on Searle approaches on Intentional mental states. The first part analyzes Archer's model apropos the debate agency/structure, and, the following section concerns the agency, taking into account the Intentional mental states. If habits and reflection, as Archer argues, depend on the morphostasis and societal morphogenesis, such processes must be manifested in consciousness somehow. This research argues that analysis of consciousness and Intentional states allow understanding the influence of the social order over the individual, specifically from beliefs and its satisfaction condition.
\end{abstract}

\section{KEYWORDS}

Agency; Beliefs; Morphogenesis; Mosphostasis; Structure. 


\section{INTRODUCCIÓN}

El problema de la agencia humana es uno de esos tópicos transdisciplinares per se; los términos estructura, cultura, agente, instituciones, roles, hábitos o creencias, recurrentes en la bibliografía sobre agencia, remiten a una gama muy amplia de disciplinas y saberes. Sea una ventaja o desventaja, lo cierto es que, más allá de la pluralidad de interpretaciones, una considerable ambigüedad rodea conceptos como estructura (Fleetwood 2008), instituciones (Camargo 2015) o cultura (Gans 2012). Aclarar los conceptos, sin embargo, no resolvería las preguntas más acuciantes sobre, por ejemplo, cómo incide la estructura sobre la agencia o cómo, paralelamente, lo hace la cultura. La propuesta de Margaret Archer (1995, 2000, 2003) avanza en la dirección adecuada a partir del modelo morfogenético; las instancias, relaciones y procesos relativos a la agencia están ampliamente desarrollados, sin embargo la expresión individual necesita mayor elaboración. En otras palabras, la aproximación dominantemente sociológica olvida que la agencia se sustantiva en el individuo, y aunque ella procura rescatar la subjetividad acentuando el papel de la reflexión interna, es necesario relacionar los estados mentales intencionales (Searle 1992) con los procesos sociales más amplios de morfogénesis y morfostasis. La estructura de la conciencia, por compleja y enigmática que pudiese ser, es el lugar desde donde se propone una lectura de la agencia, los hábitos y la reflexión.

El presente artículo parte de un axioma que no debería generar demasiada aprensión por parte de los científicos sociales: "los estados conscientes son causados en su totalidad por procesos neurobiológicos de nivel inferior con sede en el cerebro. Por ello, son causalmente reducibles a procesos neurobiológicos. No tienen ninguna vida propia por encima de la neurobiología" (Searle 2006: 148). Cualquier idea, creencia o deseo tiene necesariamente este trasfondo neurobiológico, lo cual, dígase enfáticamente, no plantea una antinomia con las explicaciones estructurales o culturales; ni, mucho menos, propone la idea de explicar fenómenos complejos del mundo social únicamente a partir de interacciones neuronales. Si las acciones humanas están permeadas por las estructuras sociales y por la cultura, no menos cierto es que las acciones dependan de la estructura misma de la conciencia. Archer (2003) reconoce la necesidad de bajar al nivel del individuo para mirar la forma como recibe las influencias de su entorno social; aquí se asume que el mejor punto de partida es analizar la estructura y funcionamiento de la conciencia. El propósito del artículo es evidenciar si existe un isomorfismo entre el lugar que le asigna Archer a los hábitos y a la reflexión, y el lugar que puedan tener a partir de la teoría de la Intencionalidad de John Searle.
La pregunta general sobre qué instancias inciden en la agencia humana está relativamente bien contestada, no solo desde Archer, sino a partir de autores clásicos como Bourdieu, Durkheim o Max Weber, quienes tallaron verdaderos pilares conceptuales a partir de las nociones de hábitos, normas o racionalización. Ahora la reflexión pendiente tiene que ver con "cuándo, dónde y bajo qué condiciones" (Archer 2010: 277) priman los hábitos o la reflexión. La pregunta es si existe un criterio para distinguir cuándo operan los hábitos, y en qué momento prevalece la reflexión interna. Es posible iniciar el estudio preguntándose cómo los atributos culturales y estructurales de una sociedad influyen en sus miembros, o, desde otro ángulo, cómo los individuos asumen las influencias para traducirlas en agencia por medio de los poderes de la persona (Archer 2003: 8).

Archer (2003) adopta esta segunda perspectiva y el presente artículo sigue su estela, a partir de los postulados sobre Intencionalidad propios de la filosofía de la mente de John Searle. La premisa básica que recibe escasa atención de Archer (2003: 10) en su crítica al "reduccionismo neurológico", es que los procesos neurales subyacen a cualquier tipo de agencia. Reconocer esto no implica, como ella supone, eliminar los poderes causales de la reflexión (Archer 2003: 11). La conciencia y la Intencionalidad tienen una base biológica y "la solución apropiada al problema mente-cuerpo reside en no negar la realidad de los fenómenos mentales, sino en apreciar adecuadamente su naturaleza biológica" (Searle 1992: 15). Dicho esto, la discusión sobre la agencia no se clausura al develar las regiones cerebrales y los procesos neurales implicados en una decisión cualquiera, como ir de compras; se trata de una "causa mecánica" de la acción, mientras las causas subjetivas (personales) y objetivas (estructurales) deberían discutirse. Como criterio de validez para dirimir el asunto de las causas estaría por ver si, de hecho, una explicación de las "causas mecánicas" se acompasa con explicaciones de corte sociológico, si se mantiene alguna coherencia entre las dinámicas de uno y otro nivel. El presente artículo avanza en esta dirección.

La importancia de los estados intencionales es su naturaleza primordial; son, por así decirlo, los bloques elementales sobre los que se constituye la conciencia, la forma como el cerebro relaciona al cuerpo con el mundo (Searle 1992: 13). Archer (2003: 8) reconoce la necesidad de observar cómo los individuos reciben las influencias de la estructura y la cultura, pues son ellos quienes a la larga determinan lo que se hace y lo que no. Analizar estados intencionales como las creencias puede ser útil para esclarecer preguntas como: ¿cuándo y por qué actuamos por hábito o reflexión interna?, ¿por qué resultan tan persistentes los hábitos?, ¿por qué coexisten hábitos y reflexión como motores de la agencia? 


\section{LA AGENCIA: UNA LECTURA DESDE MARgaret Archer}

La pregunta por la agencia involucra, al menos de forma tácita, la reflexión filosófica sobre el libre albedrío, con la diferencia de concentrarse en el predominio de factores estructurales y culturales sobre el individuo, más que en las voliciones y la libertad de la voluntad. En palabras de Searle, la interrogante es “¿cómo pueden existir genuinas acciones libres en un mundo donde todos los eventos, al menos en el nivel macro, aparentemente tienen condiciones antecedentes como causas suficientes?" (Searle 2007: 10). Desde el problema estructura/agencia el punto es si la estructura determina a la agencia o la agencia a la estructura, y si en medio de estas relaciones no terminan difuminándose estructura y agencia en una amalgama incomprensible.

Archer (2010) afirma que estructura, cultura y agentes tienen propiedades y poderes emergentes irreductibles entre sí, por tanto se plantea una distinción analítica de estas tres entidades. La autora rechaza las explicaciones que efectúan algún tipo de conflación, supeditando los poderes de los individuos a la estructura (conflacionismo descendente), o inversamente, subyugando la estructura a los individuos (conflacionismo ascendente); inclusive se critica la conflación central, donde se funden estructura y agencia (Aedo 2014) (Archer 1995). Para ella, lo que pueda entenderse como agencia estriba en las relaciones de estas tres instancias (estructura, cultura y agentes) y sus poderes causales.

Para superar las aproximaciones deficitarias, Archer construye el modelo morfogenético a partir de una línea de tiempo donde los agentes encuentran una estructura previa, y posteriormente, por medio de la interacción social, la reproducen o elaboran generando un nuevo estado estructural. El modelo morfogenético no puede ser determinista, pues la estructura en T1 fue creada por interacciones sociales previas, y la estructura reconstituida en $\mathrm{T} 4$, a su vez, será transformada o conservada por medio de futuras relaciones sociales. Pese a que en el mundo estructura y agencia operan juntas continuamente, en términos analíticos es importante distinguir entre emergencia-interacción-resultado para explicar las diferentes formas de elaboración estructural a través del tiempo (Archer 1995: 168).

"Morfogénesis se refiere a los procesos que tienden a elaborar o cambiar la forma, estructura o estado de un sistema, y morfostasis a procesos en sistemas complejos que tienden a mantenerlos inalterados" (Archer 2010: 274). La influencia de la estructura en T1 se refleja en la relación (a) en T2, no obstante los procesos de morfogénesis o morfostasis propios de la relación (b), se verán reflejados en la reproducción o elaboración estructural en T3-T4. Naturalmente, si el resultado es de morfostasis, T1 y T4 no tendrán que ser muy diferentes, en cambio en la morfogénesis T1 y T4 serán distintos y seguro iniciará un nuevo ciclo de cambios encadenados (ver figura 1). Más allá de las representaciones esquemáticas de la teoría, las preguntas más interesantes apenas están por plantearse: ¿cuándo se presenta la morfogenésis?, ¿cuándo la morfostasis?, ¿qué actores llevan a cabo estos procesos?, ¿dónde se presentan?

En el plano del agente se encuentran los motores de la acción humana estudiados por la sociología: los hábitos y la reflexión. En la línea argumental expuesta, hábitos se relaciona con morfostasis y reflexión con morfogénesis, Archer (2010) considera que la clave para sopesar la importancia de uno u otra radica en analizar las relaciones (a) y (b). La estructura preexistente en T1 determina las situaciones en las cuales acaece la interacción social, específicamente la relación (a) establece "aquello que (estructural y

Figura 1

Secuencia morfogenética básica.

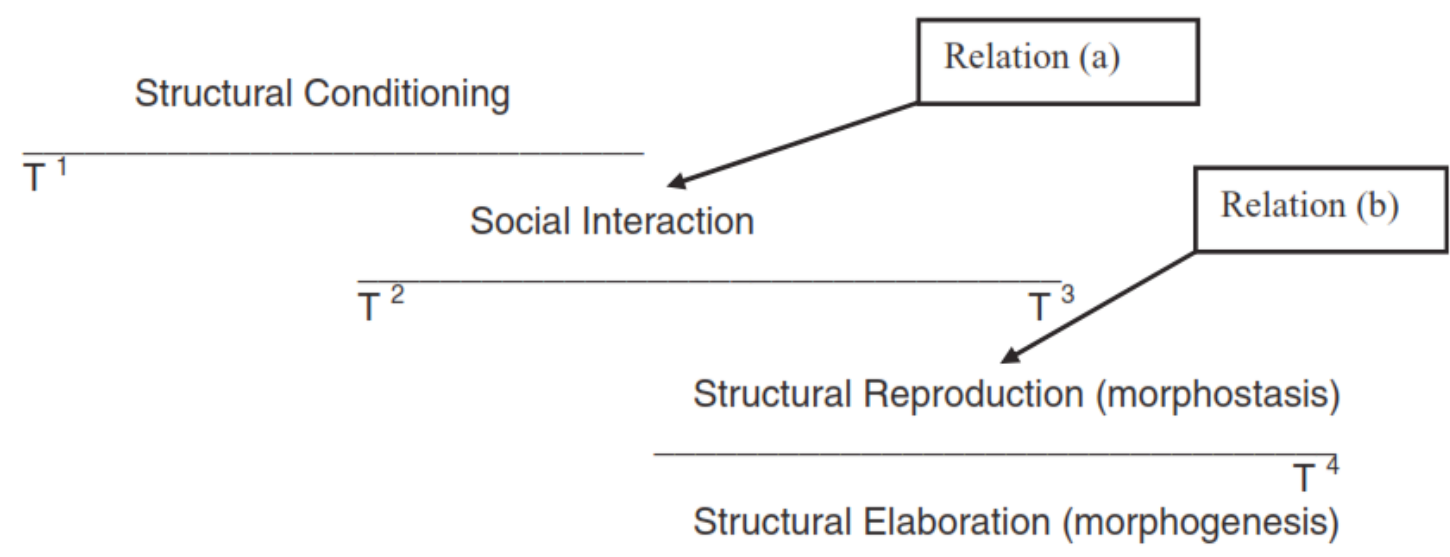

Fuente: Archer (2010: 275). 
culturalmente) ha de ser distribuido, la matriz de roles existente; la proporción de posiciones disponibles en cualquier momento y las ventajas y desventajas asociadas a ellas [...]" (Archer 1995: 201). Estos poderes propios de la estructura sociocultural se manifiestan en las relaciones sociales, de suerte que no tienen una influencia tout court; necesitan, perentoriamente, al individuo para ser funcionales. "En síntesis, la activación de restricciones y posibilidades depende de la recepción subjetiva por individuos y grupos" (op. cit.: 278).

Archer sugiere tres condiciones para el ejercicio de los poderes de restringir/permitir por parte de la instancia estructural-cultural, poderes que se manifiestan sobre los agentes: 1. La existencia de proyectos humanos; 2. Una relación de congruencia/incongruencia con los proyectos de los agentes; 3. Los agentes deben responder a estas influencias de forma condicional más que determinista, se valen de la deliberación reflexiva sobre la naturaleza de su respuesta, y sus poderes personales incluyen la habilidad para soportar o evitar su influjo (2010: 278). Las condiciones 1 y 2 permiten vincular la agencia con los estados intencionales, serán reinterpretadas y matizadas en la siguiente sección; la condición 3, en cambio, resulta problemática. Es sabido el compromiso de Archer con la reflexión interna como proceso destacado para la agencia, autores como Fleetwood (2008) y Hodgson (2004a) han remarcado la importancia de los hábitos, y han entablado un contrapunto con ella. Da la impresión de que el compromiso de Archer con la deliberación va demasiado lejos.

Afirmar la reflexión como instancia necesaria para que las influencias estructural-culturales se manifiesten en el individuo es, por decir lo menos, cues- tionable. Bourdieu señala el carácter profundo del habitus, capaz de producir estilos de vida cuyas relaciones devienen en "sistemas de signos socialmente calificados (como "distinguidos", "vulgares", etcétera)" (Bourdieu 2012: 202). Se colige que los calificativos de "distinguido" o "vulgar" no se introyectan por medio de la reflexión, sino que más bien se transmiten como "reconocimiento de un orden establecido" (Bourdieu 2012: 202). Tal vez no suceda siempre de forma automática, en algunos casos habrá reflexión y distanciamiento, pero definitivamente puede considerarse que la estructura incide sobre los individuos por medio del habitus, incluso sin mediación alguna de la reflexión.

Cuestionar la tercera condición de Archer trae a colación la pregunta, ¿cuál es el lugar de los hábitos en la agencia humana? El argumento de la autora asume que "la hegemonía del hábito depende de la morfostasis societal" (Archer 2010: 279), aquella, a su vez, depende de un bajo nivel de diferenciación contextual y un bajo nivel de diferenciación de ideas (Archer 2010: 280).

La Figura dos recoge el planteamiento de Lockwood sobre la prevalencia de morfostasis o morfogénesis según la integración social y la integración de los sistemas estructural/cultural (Archer 1995: 295). La baja diferenciación estructural y la poca diferenciación de ideas, nótese, se adecuan a lo que se puede entender grosso modo como sociedades tradicionales. El cuadrante superior izquierdo debe ser ocupado más fácilmente por sociedades simples, con incipiente división del trabajo, poca diferenciación funcional entre sus miembros y una cultura societal homogénea. De ahí se desprende que las sociedades tradicionales luzcan morfostáticas y

Figura 2

Morfostasis y morfogenésis.

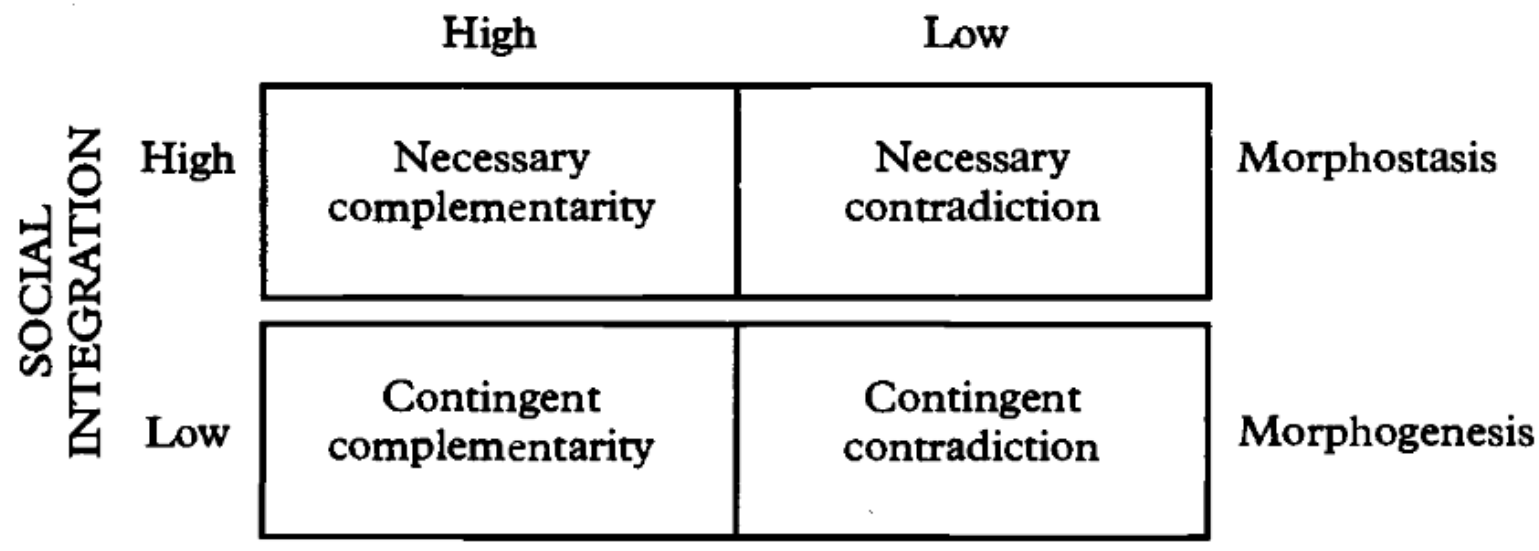

\section{SYSTEMIC INTEGRATION (structural or cultural)}


las sociedades modernas morfogenéticas. "La morfostasis cultural, a través de la reproducción constante de ideas entre una población uniforme, genera un ambiente de ideas altamente conducente al mantenimiento estructural" (Archer 2010: 280). No sobra añadir que, de forma análoga, el mantenimiento rígido de los roles y las posiciones jerárquicas coadyuva con la preservación de la cultura.

La contracara evidente de lo anterior es que la reflexión depende de la morfogénesis. La innovación, las ideas originales y la pluralidad de opciones propia de la vida moderna hacen necesaria la reflexión de los agentes. Por supuesto, la diversificación de la cultura desafía la integración con las disposiciones estructurales, y la alternativa lógica para los actores es reflexionar. "En lugar de la guía de los hábitos, los sujetos se vuelven cada vez más dependientes de sus preocupaciones personales como su única guía de acción" (Archer 2010: 285). Aun aceptando la explicación de reflexión y hábitos de acuerdo con los procesos societales de morfogénesis y morfostasis, no se ha especificado el mecanismo por el cual el individuo internamente sigue esta dinámica social más amplia, es decir, en qué medida, mientras los individuos determinan en última instancia la agencia (como Archer misma reconoce), la morfostasis y morfogénesis se traduce a nivel mental. Tal vez por esta carencia continúan las discusiones con autores como Fleetwood (2008), Elder-Vass (2007), Sayer (2005) o Hodgson (2004a, 2004b), quienes reivindican un lugar para rutina y hábitos en tanto motores de acción.

La siguiente sección pretende analizar la primacía de hábitos o reflexión desde una perspectiva interna. Se pregunta de qué forma un estado mental intencional como las creencias puede ser crítico para traducir la morfostasis/morfogénesis social, en hábitos o reflexión.

\section{LAS CREENCIAS COMO ESTADOS INTENCIONALES PRIMORDIALES}

Los estados intencionales son representaciones mentales sobre algo, o dirigidas a objetos o estados de cosas del mundo (Searle 1992). Las creencias, como explica Searle, son sobre algo, se entienden aquí como una atribución de características a cualquier cosa o idea. "Los extraterrestres existen" es una creencia, como también lo sería creer que "la sangre es roja"; se atribuye el carácter de "ser existente" al extraterrestre y el color rojo a la sangre, independientemente de la verdad que entrañen estos enunciados. El rasgo definitorio de las creencias no es su correspondencia con la realidad, sino su direccionalidad en tanto estado intencional. Hay estados intencionales como los deseos que tienen intencionalidad en la medida en que deseamos algo; o las intenciones, entendidas como estado intencional, se definen como la voluntad de hacer algo. En ese sentido, debe quedar absolutamente claro que las creencias no implican la "intención de hacer", la intención es un estado intencional relacionado con las creencias, pero distinto a ellas.

Los estados intencionales tienen un efecto causal en los agentes, lo que Searle denomina causación intencional (Searle 1992: 123). Si bien pocos afirmarían seriamente que lo que pensamos está desconectado de lo que hacemos, la teoría causal predominante de raigambre humeana no se adecúa bien a los estados intencionales. Según Hume, el aspecto central para identificar una relación causal es la unión constante entre causa y efecto, entendiendo, además, que la causa produce siempre el mismo efecto, y el mismo efecto no surge nunca más que de la misma causa (Hume 2001: 138). Cuando se presenta un estado intencional que conlleva una acción, no necesariamente se cumple la condición de Hume, aun cuando la causalidad es obvia para el agente. Por ejemplo, un hombre que enciende un cigarrillo mientras espera que un familiar salga de cirugía puede saber que decidió fumar por un estado de ansiedad, lo que no significa que el estado de ansiedad siempre cause la acción de fumar; ni siquiera podría garantizar el hombre que en otro episodio de ansiedad volverá a hacerlo. Como realista causal, Searle (1992:130) interpreta la causalidad intencional como un asunto de vivencia, como una conciencia inmediata, que no implica una regularidad de eventos ni se compromete con alguna ley universal.

En la medida en que existen diversos estados intencionales, ¿por qué erigir el análisis desde las creencias y no desde la intención, si esta última parece vincularse más directamente con la agencia? La intención, como estado intencional, tiene que ver con los "proyectos humanos", la primera condición propuesta por Archer para el ejercicio de los poderes estructural-culturales sobre el agente. Aquí se considera la creencia un estado intencional primordial, en el sentido de que precede a la intención, es decir, querer hacer $X$ requiere una previa atribución de características a $\mathrm{X}$, o, de otra manera, una concepción de X. Un niño de cinco años no puede tener la intención de realizar una consignación bancaria, ya que no puede representarse mentalmente la consignación bancaria, no sabe qué es. "Los estados intencionales no funcionan de forma independiente o atómica, sino que cada uno de ellos tiene su contenido y determina sus condiciones de satisfacción únicamente con relación a muchos otros estados intencionales" (Searle 1992:150). Significa que la intención de efectuar una consignación bancaria depende de creencias sobre lo que es una consignación y lo que es un banco, además del procedimiento concreto para realizar la transacción y cualquier cantidad de estados intencionales conexos que conforman una Red (networking).

Trabajos como el de Krause interpretan la agencia desde la forma de cómo los actores se involucran en 
la intención colectiva de $\mathrm{X}$, aunque $\mathrm{X}$ no esté en sus intereses (2012: 323). Los estudios enfocados de este modo no apuntan a la cuestión más fundamental de X como estado intencional. Los economistas institucionales han discurrido emocionados sobre las instituciones, entendidas como restricciones y oportunidades que moldean las interacciones humanas (North 1991), o como "sistema de reglas sociales establecidas e instaladas que estructuran las interacciones sociales" (Hodgson 2006: 18). Sin embargo, permanecen en el nivel epidérmico pues las restricciones y oportunidades se dirigen a las intenciones, pero ellas mismas se incardinan en creencias, y estas últimas se ven afectadas por los planos simbólico (cultural) y estructural, los cuales deben separarse con fines heurísticos (Camargo 2015) (Portes 2006).

Aceptemos que los estados intencionales son dirigidos (aunque algunos no lo sean), esa dirección de ajuste se entiende como una forma de relacionarse con el mundo (Trigos 2010: 93). La dirección de ajuste de las creencias procede de la mente hacia el mundo (mente-mundo), se espera que lo que creo de $X$, en efecto sea $X$. Las condiciones de satisfacción, como segunda característica de los estados intencionales (dirigidos), "son aquellas condiciones que, en tanto que determinadas por el contenido intencional, deben darse si el estado se satisface" (Searle 1992: 28). La creencia "los extraterrestres existen" incluye su condición de satisfacción, que los extraterrestres efectivamente existen, aunque el hecho de no haberse comprobado la existencia de ellos no destruye la creencia misma ¿Cómo es eso posible?

Las condiciones de satisfacción son el punto más importante de la argumentación venidera, por ello conviene añadir algo. La creencia no depende de una verificación empírica de su contenido, digamos que si una comunidad cerrada considera que los extraterrestres existen, para ellos, definitivamente, existen. En este caso se cumple la condición de satisfacción, no obstante, supóngase una pluralidad de comunidades, unas piensan que existen los extraterrestres y otras que no, en este caso no se satisface la creencia sobre la existencia, aunque no por ello desaparece. Hoy en día las teorías conspirativas subsisten aunque no tengan un respaldo mayoritario. La imposibilidad de verificación empírica y la ausencia de consenso tienen efectos críticos como se verá más adelante.

En cierta forma las creencias se pueden satisfacer desde la experiencia del individuo y sus percepciones, esto es importante, pero en un sentido de sociedad la condición de satisfacción se cumple cuando existe consenso generalizado de que algo es así, independientemente de la correspondencia con la realidad. Este es el caso de los mitos en las sociedades tradicionales cerradas. Cuando entran en juego grupos que discrepan, la creencia no se satisface y en consecuencia quienes estaban convencidos de algo ahora pueden cambiar su creencia o defenderla. La defensa implica una toma de posición frente a quienes no están de acuerdo, y puede, a su vez, requerir de la búsqueda de pruebas para robustecer la creencia inicial, e incluso convencer a los detractores.

En el individuo este proceso puede ser matizado por una "instancia de verificación" de las creencias. La instancia de verificación se entiende como un cuerpo de ideas, más o menos generalizado respaldado por un consenso social, capaz de explicar gran cantidad de asuntos del mundo. La condición de satisfacción de las creencias puede cumplirse si la instancia de verificación (sea religión o ciencia) estipula algo sobre la creencia particular. Religión y ciencia, como instancias de verificación, tienen diferencias clave: la primera es holista, se basa en el dogma y tiende a la permanencia; la segunda es parcial, se basa en la evidencia y tiende al cambio.

Esto significa, en efecto, que la religión satisface muchas creencias (aunque no se verifiquen empíricamente), mientras que la ciencia solo satisface algunas creencias verificadas. Sin entrar en consideraciones epistemológicas mayores, se reconoce el estatuto especial de la ciencia en tanto verifica creencias más allá del ámbito social donde se produce el conocimiento. No es lo mismo decir "creo que en un plano la distancia más corta entre dos puntos es una línea recta", a decir, "creo que los hombres fueron creados por los dioses a partir del maíz". Aunque en principio ambas afirmaciones pueden ser verdaderas o falsas hasta ser verificadas, la primera es falsable (Popper 1991) incluso empíricamente, mientras que la segunda no. Así, la primera creencia puede ser entendida por un universo mucho más amplio y la segunda depende íntimamente del contexto donde surgió. Es comprensible por qué la primera afirmación es cierta a partir de un experimento sobre una hoja de papel, mientras que en la segunda no vale la pena emprender una refutación pues no es falsable.

Que la ciencia cumpla las condiciones de satisfacción de una creencia a partir de la verificación (correspondencia de la creencia con el mundo demostrada a través del método científico) no significa que produzca simplemente creencias en sentido vulgar, o que el conocimiento científico tenga exactamente el mismo valor y utilidad que, por ejemplo, el conocimiento basado en la magia y adivinación. Para no desviar la discusión hacia la epistemología, solo debe tenerse en cuenta por ahora que se distinguen dos instancias de verificación por la forma como cumplen la condición de satisfacción de las creencias, a partir de la verificación o sin ella. La ciencia, dado que no puede estudiar infinidad de asuntos, no se preocupa por explicar la totalidad de ideas y creencias; esto permite que las condiciones de satisfacción no se cumplan y surja la reflexión. La religión, o incluso las doctrinas e ideologías holistas, proponen un esquema explicativo general que procura cumplir las condiciones de satis- 
facción de la mayoría de creencias, de ahí que sean funcionales a la morfostasis y los hábitos.

La tesis del artículo sugiere que mientras se cumpla la condición de satisfacción de las creencias, surgirán hábitos de acuerdo con el contenido particular de la creencia, en cambio, cuando no se satisface, se abrirá paso a la reflexión, bien sea para cuestionar la creencia propia y abandonarla, o para establecer una línea argumental que la defienda. La satisfacción de la creencia implica dar como ciertas las características de $\mathrm{X}$, y de esa forma puedo tener la intención (en este momento sí cobra relevancia la intención) para actuar conforme con los atributos adjudicados a X. Un hábito surge, entonces, cuando se satisface una creencia; por decirlo así, cuando se convierte en algo verdadero, verificado o no. Comoquiera que la ciencia no satisface todas las creencias deja campo suficiente para la reflexión. Puntualmente, los hábitos en las sociedades abiertas, donde la ciencia prima como instancia de verificación, surgen a partir de creencias verificadas a través del método científico, especialmente eficaces por sus efectos prácticos (tecnología). Por dar un ejemplo trivial, nadie reflexiona en las mañanas sobre las propiedades de la pasta dental, y si en realidad sirve para la higiene bucal, las personas se cepiIlan los dientes de forma automática, por hábito, sí, pero más importante aún, por la satisfacción verificada de la siguiente creencia: "la pasta dental es necesaria para la higiene bucal".

El anterior ejemplo no sugiere que antes de efectuar una acción rutinaria surja en la conciencia la creencia verificada que la sustenta, estos contenidos fueron interiorizados de tal manera que se integran al trasfondo preintencional (Searle 1992, 1997) y por eso mismo su efecto es automático e inconsciente. La pervivencia de los hábitos y su reluctancia al cambio puede deberse, desde el nivel individual, al hecho de que algunas creencias pierden su carácter intencional cuando se inscriben en el trasfondo. Sería muy extraño que alguien pensara todas las mañanas antes de lavarse los dientes en las bondades de la pasta dental, el hábito simplemente está ahí. Es presumible que las creencias sedimentadas en el trasfondo sean funcionales para reducir el costo cognitivo y estructurar la experiencia, las categorías simples de "esto es una persona" o "esto es un vestido" (Searle 1997:145) son ejemplos de contenidos de trasfondo que permiten navegar en el mundo.

Las categorías del cuadro son tipos ideales weberianos más que descripciones y, en la práctica, los agentes tienen creencias en los tres estados; también existen varias instancias de verificación en una sociedad. Cerrada y abierta solo alude al contacto de una sociedad con otra, si en ella existe diversidad de ideas o no; la reflexión de esta manera parece estar en función de la complejidad social. Los hábitos no desaparecen por el costo cognitivo, analizar cada movimiento y actividad nos llevaría a la parálisis. Para evitar la hiperreflexión se realizan cantidad de actos mecánicamente, dando valor a las creencias que subyacen a ellos por experiencia, por apelación inmediata a la instancia de verificación, o incluso porque esas creencias han sido integradas en el trasfondo. Nótese que el preámbulo "los científicos determinaron que X produce ciertos efectos", tan común en la publicidad, pretende que las personas den por verificada una creencia para que adquieran un producto o asuman determinadas actitudes de forma acrítica. Algunas de las relaciones más importantes pueden entenderse mejor por medio de la siguiente fórmula de la agencia:

$$
\begin{aligned}
& \mathrm{A}=(\mathrm{EC})^{*}(\Delta \mathrm{E}+\Delta \mathrm{S}) \\
& \mathrm{A}=\text { Agencia } \\
& \mathrm{EC}=\text { Estado de la creencia } \\
& \Delta \mathrm{E}=\text { Cambios en la estructura social } \\
& \Delta \mathrm{S}=\text { Cambios en el nivel simbólico }- \text { cultural }
\end{aligned}
$$

La fórmula establece una relación entre los estados de las creencias (satisfecha sin verificar, no satisfecha y satisfecha verificada) y los cambios en la estructura social y la cultura. Entre las creencias y los niveles social y simbólico se utiliza la multiplicación en lugar de la adición, pues ninguna de las partes

Tabla 1

Estado de las creencias, instancias y proceso de agencia.

\begin{tabular}{|c|c|c|c|c|}
\hline Estado de la creencia & $\begin{array}{c}\text { Tipo de sociedad } \\
\text { (nivel estructural) }\end{array}$ & $\begin{array}{c}\text { Instancia de } \\
\text { verificación } \\
\text { (nivel simbólico- } \\
\text { cultural) }\end{array}$ & Proceso social & Proceso del agente \\
\hline $\begin{array}{c}\text { Satisfecha, sin } \\
\text { verificar }\end{array}$ & Tradicional cerrada & Religión & Morfostasis & Hábitos \\
\hline Insatisfecha & Tradicional abierta & Religión y sectas & $\begin{array}{c}\text { Morfostasis } \\
\text { y progresiva } \\
\text { morfogénesis }\end{array}$ & $\begin{array}{c}\text { Hábitos y alguna } \\
\text { reflexión }\end{array}$ \\
\hline Satisfecha verificada & $\begin{array}{c}\text { Moderna } \\
\text { (abierta) }\end{array}$ & Ciencia & $\begin{array}{c}\text { Morfogénesis y limitada } \\
\text { morfostasis }\end{array}$ & $\begin{array}{c}\text { Reflexión y algunos } \\
\text { hábitos }\end{array}$ \\
\hline
\end{tabular}


puede tomar un valor 0 , esto anularía la agencia. Por obvio que parezca, vale anotar que la imposibilidad de creencias producto de un trastorno neurológico grave hace imposible cualquier agencia humana, la fórmula debe expresar estas realidades. Los cambios en la estructura social y en el nivel simbólicocultural se pueden operar por medio de la adición $(\Delta \mathrm{E}+\Delta \mathrm{S})$ ya que, en la práctica, alguno de los dos puede alterarse mientras el otro permanece constante. Los fenómenos de colonización y descolonización son un buen ejemplo donde los cambios estructurales políticos y económicos no se corresponden con los cambios culturales, generando tensiones y traumatismos sociales.

Acorde con lo dicho al principio, se mantiene la prioridad del individuo y sus creencias, pues sin tales estados intencionales sería imposible la acción. Los poderes causales de la estructura social y la cultura únicamente pueden ejercerse a partir de su impacto sobre el estado de las creencias, y no simplemente actuando sobre "los proyectos humanos" (Archer 2010) o "las intenciones" (Krause 2012). De forma sutil, inclusive, se pueden promover creencias que a la larga inciden en la agencia sin ninguna necesidad de afectar de manera explícita los proyectos humanos. Como ya se mencionó, el monismo favorece la morfostasis y produce hábitos, mientras el pluralismo permite la morfogénesis y abona el terreno para la reflexión de los individuos. $\Delta \mathrm{E}$ y $\Delta \mathrm{S}$ pueden aumentar las posibilidades de la agencia cuanto mayores sean sus valores, de ahí que sociedades modernas, caracterizadas por el pluralismo y los hallazgos científicos permanentes, resultan altamente morfogenéticas, mientras que las sociedades cerradas, sin apenas cambios, resultan morfostáticas, y sus miembros parecen actuar de manera más homogénea, mecánica y ritualizada.

La fórmula de la agencia debería en realidad incluir la intención como estado intencional: el hecho de que alguien haga algo colige que tuvo la intención de hacerlo (por lo menos en los casos de acción voluntaria), si hago $\mathrm{X}$ obtengo $\mathrm{Y}$. Sin embargo, por cuestiones de extensión y complejidad se parte de la creencia, pues el deseo o la intención requieren alguna noción de lo que son (o creo que son) $X$ e $Y$, además que entiendo como $X$ me puede llevar a $Y$ (Searle 1992). Aunque no todas las creencias pueden resultar en una intención, sin ellas es imposible la agencia y considero que son el estado intencional desde donde debe iniciarse un análisis como este. Otra razón para eludir la intención como punto de partida es que algunos estados intencionales cuya sede es el inconsciente reprimido o el inconsciente dinámico, pueden actuar de forma causal en el comportamiento humano (Searle 2006: 298) y la manera como sucede esto aún está por descifrarse.

La fórmula solo es un recurso pedagógico para expresar de manera sintética ciertas relaciones con- ceptuales, en la práctica ninguno de los componentes podría tomar un valor 0 . De hecho, siempre habrá creencias y algunos cambios, aunque sean pequeños, en las condiciones estructural-culturales. Se concluye por ahora que parece existir una relación directa entre la complejidad social y la necesidad de reflexión. El individuo en un ambiente complejo tendrá que evaluar cómo desenvolverse, aunque grandes porciones de su mundo se rijan por hábitos interiorizados como normas de cortesía, normas de tránsito implícitas, rutinas de higiene, etc.

\section{AlgunAs RESPUESTAS PROVISIONALES}

Se han presentado, tanto el modelo morfogenético de Archer como las dinámicas de las creencias, en su calidad de estados intencionales con una condición de satisfacción que puede cumplirse o no. En una línea que transita desde la "continuidad contextual" de las sociedades tradicionales, pasando por la "discontinuidad contextual" de las sociedades modernas, hasta la "incongruencia contextual" propia de las últimas dos décadas del siglo XX (Archer 2010: 296-297), puede entenderse el despliegue de la reflexión y el ocaso de los hábitos. "Esta secuencia está internamente relacionada con un incremento del alcance y el rango de la reflexividad, dado el aumento de situaciones novedosas en el orden social, donde los sujetos no pueden depender de la acción rutinaria como guía apropiada de la acción" (Ibíd.: 297).

Esta conclusión de Archer puede sustentarse a partir del análisis previo de la creencia como estado intencional y las subsecuentes condiciones de satisfacción. Frente a la pregunta sobre ¿cuándo y por qué actuamos por hábito o reflexión?, se sugieren dos aspectos cruciales: primero, un contexto más complejo, variopinto y plural, en una palabra, abierto, produce insatisfacción de las creencias establecidas (confrontación con creencias distintas) y por ende mayor reflexión; segundo, en la medida en que hay una o varias instancias de verificación (ciencia y religión) siempre existirán creencias lo suficientemente satisfechas como para producir hábitos (sea lavarse los dientes u orar tres veces al día). El coste cognitivo de la reflexión es sustancial para explicar por qué perduran los hábitos, ellos sirven como atajos para producir continuidad contextual, dejando la reflexión para dirimir asuntos donde las condiciones de satisfacción no se cumplen de forma generalizada. El trasfondo también resulta esencial para generar hábitos al condensar creencias satisfechas en categorías estructurales del mundo.

Vale recordar que lo propuesto no es una regla o una ley que invariablemente deba acaecer, la complejidad del contexto solo podrá tener efectos en los individuos cuando se divulgan nuevas ideas. Algunas creencias, como se mencionó, no tienen ninguna incidencia sobre la agencia, pero aquellas que soportan 
estados intencionales como los deseos e intenciones son muy importantes. Una vez las creencias entran en el horizonte vital de los individuos se crean las condiciones de posibilidad de la reflexión. El ejemplo sobre el cual discuten Fletwood (2008) y Archer (2010) es revelador: Angie, siguiendo a su madre, a su tía y a varios amigos de la familia se emplea como secretaria (Archer 2010: 300). Fletwood afirma que Angie no habría considerado seriamente trabajar como soldadora. En realidad ella ni siquiera habría discutido con su familia y amigos la posibilidad de ser soldadora, la idea no se encuentra en "la pantalla del radar" de Angie (Fletwood 2008: 18) y por eso no puede contemplarla. Supóngase que alguna amiga de Angie trabajara como soldadora, en ese caso Angie lo habría contemplado, pero el hecho de no tener la creencia de que las mujeres trabajan habitualmente como soldadoras hace esa posibilidad inexistente para ella.

En esta instancia es claro que no debe plantarse un dualismo entre hábitos (Hodgson 2004a) (Fletwood 2008) y reflexión (Archer 2003), ambos son necesarios y su protagonismo varía según se describió anteriormente. Existe un sesgo epistemológico que juega en contra de los hábitos como objeto de estudio: piénsese de todas las actividades diarias cuáles se desarrollan a partir de hábitos y cuáles a partir de la reflexión. Resulta que los hábitos actúan en momentos aparentemente irrelevantes (saludar, despedirse, dar las gracias, bañarse, lavarse los dientes etc.,) mientras que la reflexión entra en juego en momentos más importantes. Es probable que este hecho tan simple permanezca en el trasfondo de los argumentos de Archer en pro de la reflexión. Aunque procesos más automáticos como las intuiciones pueden ser motores importantes de la acción (Haidt 2001), aún es necesario explicar cómo estados mentales no conscientes pueden afectar a la agencia, por no mencionar el papel del inconsciente que debe ser esclarecido de una manera más robusta científicamente de lo que lo hiciera Freud (Searle 2006: 299) (Popper 1991a: 59-60).

De hecho, múltiples asuntos relacionados con los estados intencionales y la agencia permanecen en las sombras. Se ha dicho que la condición de satisfacción de las creencias incide en la agencia a la hora de producir hábitos o reflexión, no obstante la relación de las creencias con estados intencionales como la intención y el deseo aún no se ha caracterizado, ni será posible hacerlo en este momento. Los "proyectos humanos" a los que aludía Archer son, en otras palabras, estados intencionales como la intención y el deseo, los cuáles se basan en creencias, pero plantean relaciones mucho más complejas. No se trata ahora de la atribución de características, sino de elegir un curso de acción para lograr un objetivo. En este caso debe articularse la racionalidad como principio organizador de una forma que no se ha explorado en este artículo.

\section{Conclusiones}

La idea que ha sustentado el artículo es que hábitos y reflexión dependen de las creencias de las personas, puntualmente si se cumple la condición de satisfacción o no. Cuando una persona cree firmemente en la veracidad de sus creencias tiende a actuar por hábito, las comunidades tradicionales estudiadas por etnógrafos resultan plétoras de rituales y hábitos, viven sus vidas de acuerdo a su religión y costumbres. Todo aquel que haya hablado con una persona profundamente religiosa notará la infausta tarea de hacer que cambie de opinión; en alguna ocasión pude ver cómo alguien analizaba la pugna política entre izquierda y derecha como un evento fundamentalmente explicado por la biblia. Cuando existe una respuesta "para todo" basta apelar a la instancia de verificación y actuar de acuerdo con ella. Los cambios en $\Delta \mathrm{E}$ y $\Delta \mathrm{S}$ desafían las creencias, avivan las tensiones, producen discontinuidad contextual, para las sociedades tradicionales son un desafío o acaso un trauma, para las sociedades modernas son moneda corriente.

La cuestión es cómo se enfrentan quienes dan por ciertas sus creencias e ideas a un mundo interconectado, a un mundo donde algunos no solo piensan distinto a ellos, sino de forma radicalmente opuesta. ¿Es posible simplemente apelar a la instancia de verificación para satisfacer las creencias? Resulta muy difícil cuando las instancias son tan variadas, no se trata de escoger religión, el hecho es que las más disímiles ideas y filosofías están al alcance de todos merced a las tecnologías de la información, ergo el autoritarismo es el camino expedito para contener la reflexión ¿Podrán las personas transitar de la morfostasis societal y la acción guiada por hábitos, hacia una agencia reflexiva que les permita insertarse en un contexto globalizado altamente morfogenético? A juzgar por fenómenos actuales como las guerras en Medio Oriente y el discurso de actores como ISIS, este proceso no resulta para nada sencillo, aunque más desconcertante aún es que ciudadanos acostumbrados al pluralismo de ideas y al cambio encuentren atractivo el proyecto de grupos que esencialmente propenden a la morfostasis y el control.

Se ha dicho que hábitos y reflexión dependen de las creencias, ambos son necesarios y persistentes. El problema no es que primen los hábitos o la reflexión, la cuestión es en qué momento, si en asuntos que atañen a la "estructura básica de la sociedad" (Rawls 2004: 17) se va a excluir la reflexión mientras otros deciden. Si la administración de justicia será conforme a la costumbre, si perduran la lapidación, la mutilación genital femenina o la amputación, como de hecho sucede en algunos lugares. En un mundo donde aislarse deviene en utopía y romanticismo, la cuestión es si las sociedades más tradicionales abrirán espacios 
para reflexionar sobre sus costumbres y tradiciones; no basta afirmar el relativismo de la cultura y la moral en un mundo donde las personas migran masivamente hacia sociedades abiertas, prefiriendo la incertidumbre y el desarraigo en el exilio a la opresión en su tierra.

\section{ReferenCIAs BibLIOgRÁficas}

Aedo, A. 2014. "El doble juego mutuo entre agencia y estructura en la obra de Margaret Archer: conversación interna, proyecto y fricción". Tesis doctoral. Santiago de Chile: Departamento de Sociología, Universidad Alberto Hurtado.

Archer, M. S. 1995. Realist social theory: The morphogenetic approach. Cambridge: Cambridge University Press. https://doi.org/10.1017/CBO9780511557675

Archer, M. S. 2000. Being human: The problem of agency. Cambridge: Cambridge University Press. https://doi. org/10.1017/CBO9780511488733

Archer, M. S. 2003. Structure, agency and the internal conversation. Cambridge: Cambridge University Press. https://doi.org/10.1017/CBO9781139087315

Archer, M. S. 2010. "Routine, Reflexivity, and Realism". Sociological Theory 28 (3): 272-303. https://doi. org/10.1111/j.1467-9558.2010.01375.x

Bourdieu, P. (2012). La distinción: criterio y bases sociales del gusto. Madrid: Taurus.

Camargo, A. M. 2015. "Cultura e instituciones: ¿una relación indisoluble?" Revista de Economía del Caribe 16: 162177. https://doi.org/10.14482/ecoca. 16.8082

Elder-Vass, D. 2007. "For emergence: refining Archer's account of social structure". Journal for the Theory of Social Behaviour 37 (1): 25-44. https://doi.org/10.1111/j.14685914.2007.00325.x

Fleetwood, S. 2008. "Structure, institution, agency, habit, and reflexive deliberation". Journal of Institutional Economics 4 (2): 183-203. https://doi.org/10.1017/ S1744137408000957

Gans, H. J. 2012. "Against culture versus structure”. Identities 19 (2): 125-134. https://doi.org/10.1080/107028 9X.2012.672850

Haidt, J. 2001. "The emotional dog and its rational tail: a social intuitionist approach to moral judgment". Psychological review 108 (4): 814-834. https://doi.org/10.1037/0033$295 X .108 .4 .814$

Hodgson, G. M. 2004a. "Reclaiming habit for institutional economics". Journal of economic psychology 25 (5): 651 660. https://doi.org/10.1016/j.joep.2003.03.001

\section{Agradecimientos}

Gracias a la beca doctoral otorgada por Colciencias en convenio con la Universidad del Norte fue posible realizar la presente investigación. Se extiende un reconocimiento especial a estas instituciones.

Hodgson, G. M. 2004b. The evolution of institutional economics. London: Routledge. https://doi. org/10.4324/9780203300350

Hodgson, G. M. 2006. "What are institutions?" Journal of Economic Issues 40 (1): 1-25. https://doi.org/10.1080 /00213624.2006.11506879

Hume, D. 2001. Tratado de la naturaleza humana. (Recuperado de http://www.dipualba.es/publicaciones/)

Krause, J. 2012. "Collective Intentionality and the (Re) Production of Social Norms The Scope for a Critical Social Science". Philosophyofthe SocialSciences42(3): 323-355. https://doi.org/10.1177/0048393111399100

North, D. 1991. "Institutions". Journal of Economic perspectives 5 (1): 97-112. https://doi.org/10.1257/jep.5.1.97

Popper, K. R. 1991. "Los dos significados de falsabilidad". Revista de filosofía 5: 3-12.

Popper, K. R. 1991a. Conjeturas y refutaciones: el desarrollo del conocimiento científico. Paidós Ibérica.

Portes, A. 2006. "Instituciones y desarrollo: una revisión conceptual". Cuadernos de Economía 25 (45): 13-52.

Rawls, J. 2004. Teoría de la justicia. México D.F.: Fondo de Cultura Económica.

Sayer, A. 2005. The moral significance of class. Cambridge: Cambridge University Press. https://doi.org/10.1017/ CBO9780511488863

Searle, J. 1992. Intencionalidad. Un ensayo en la filosofía de la mente. Madrid: Tecnos.

Searle, J. 1997. La construcción de la realidad social. Barcelona: Paidos.

Searle, J. 2006. La mente: una breve introducción. Bogotá: Grupo Editorial Norma.

Searle, J. 2007. Freedom and neurobiology. Reflections on Free Will, Language and Political Power. Nueva York: Columbia University Press.

Trigos, L. M. 2010. "Significado e intencionalidad". Forma y Función 23 (1): 89-99.

ANTONIO MIGUEL CAMARGo es Politólogo de la Universidad del Rosario (Colombia) y Magíster en Estudios Políticos e Internacionales. Actualmente se encuentra cursando el doctorado en Ciencias Sociales de la Universidad del Norte, en calidad de becario Colciencias. Sus áreas de interés son la ética, la psicología moral y la teoría social. 